\title{
Radioactive iodine in differentiated thyroid cancer: a national database perspective
}

\author{
Ryan K Orosco1, Timon Hussain1, Julia E Noel2, David C Chang33, Chrysoula Dosiou4, Erik Mittra5, Vasu Divi² and \\ Lisa A Orloff ${ }^{2}$
}

'Division of Head and Neck Surgery, Department of Surgery, University of California San Diego, San Diego, California, USA 2Division of Head and Neck Surgery, Department of Otolaryngology, Stanford University, Palo Alto, California, USA

${ }^{3}$ Codman Center for Clinical Effectiveness in Surgery, Department of Surgery, Harvard Medical School, Boston, Massachusetts, USA

${ }^{4}$ Division of Endocrinology, Department of Medicine, Stanford University, Stanford, California, USA

${ }^{5}$ Division of Nuclear Medicine and Molecular Imaging, Department of Radiology, Stanford University, Stanford, California, USA

Correspondence should be addressed to J E Noel: jtomlin2@stanford.edu

\begin{abstract}
Radioactive iodine (RAI) is a key component in the treatment of differentiated thyroid cancer. RAI has been recommended more selectively in recent years as guidelines evolve to reflect risks and utility in certain patient subsets. In this study we sought to evaluate the survival impact of radioactive iodine in specific thyroid cancer subgroups. Nationwide retrospective cohort study of patients using the National Cancer Database (NCDB) from 2004 to 2012 and Surveillance, Epidemiology, and End Results (SEER) database from 1992 to 2009 examining patients with differentiated thyroid cancer treated with or without RAI. Primary outcomes included all-cause mortality (NCDB and SEER), and cancer-specific mortality (SEER). Cox multivariate survival analyses were applied to each dataset, and in 135 patient subgroups based on clinical and non-clinical parameters. A total of 199,371 NCDB and 77,187 SEER patients were identified. RAI was associated with improved allcause mortality (NCDB: RAI hazard ratio (HR) 0.55, $P<0.001$; SEER: HR 0.64, $P<0.001$ ); and cancer-specific mortality (SEER: HR $0.82, P=0.029$ ). lodine therapy showed varied efficacy within each subgroup. Patients with high-risk disease experienced the greatest benefit in all-cause mortality, followed by intermediate-risk, then low-risk subgroups. Regarding cancer-specific mortality, radioactive iodine therapy was protective in high-risk patients, but did not achieve statistical significance in most intermediate-risk subgroups. Low-risk T1a subgroups demonstrated an increased likelihood of cancer-specific mortality with iodine therapy. The efficacy of RAI in patients with differentiated thyroid cancer varies by disease severity. A negative cancer-specific survival association was identified in patients with T1a disease. These findings warrant further evaluation with prospective studies.
\end{abstract}

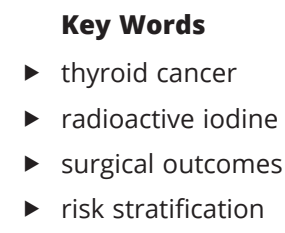

Endocrine-Related Cancer (2019) 26, 795-802

\section{Introduction}

Thyroid cancer is the most common new malignancy diagnosed in the United States. It also carries an expanding global disease burden, in part due to the increased detection of tumors that may otherwise remain asymptomatic and not impact mortality (Vaccarella et al. 2016).
In 2019 , we are likely to see nearly 57,000 new cases, and approximately 2000 deaths from this disease in the United States alone (Siegel et al. 2017). The vast majority of these cases are well-differentiated thyroid carcinomas (DTC), consisting of papillary and follicular histology, 
which carry an overall excellent prognosis, particularly in early-stage disease. With appropriate treatment, 5-year survival exceeds 95\% (Sherman 2003).

Surgery is often the first step in successful therapy for thyroid cancer. In the past, nearly all patients received adjuvant RAI; however, guidelines have recently shifted toward a more individual and stratified approach. Today, only select cases of DTC warrant RAI, typically indicated in thyroid remnant ablation, adjuvant therapy, or treatment of known persistent disease. In the 2015 American Thyroid Association guidelines for the management of thyroid cancer, RAI adjuvant therapy is routinely recommended after total thyroidectomy for patients with high-risk disease and should be considered for intermediate-risk disease (Haugen et al. 2016). It is not recommended for patients with low-risk disease (Haugen et al. 2016). The evidence supporting RAI in high-risk patient subgroups is relatively undisputed (Jonklaas et al. 2006). In the intermediate-risk DTC subgroups, there is controversy surrounding appropriate use. A growing body of literature indicates that the risk/benefit ratio is in favor of avoiding RAI in low-risk populations.

To facilitate evidence-based decision making, we sought to evaluate the association between RAI and mortality across all patients with DTC, as well as in specific subgroups. A deeper understanding of the impact of RAI therapy in DTC subpopulations may help refine its indications and reduce unnecessary treatment, cost, and adverse effects.

\section{Materials and methods}

This retrospective study included patients in the National Cancer Database (NCDB) from 2004 to 2012 and Surveillance, Epidemiology, and End Results (SEER) database from 1992 to 2009 who had DTC as their only malignancy. Histopathology inclusion criteria were papillary or follicular carcinomas (ICD-O-3 C73.9; histology 8050, 8260, 8330, 8331, 8332, 8335, 8340, $8341,8342$, or 8343$)$.

Race categories as defined by SEER were grouped as white, black, Asian, Hispanic, and other/unknown. Age was recorded at the time of diagnosis. Primary tumor, nodal, and metastatic characteristics were used to manually stage SEER patients using the 2010 American Joint Committee on Cancer (AJCC) 7 TNM criteria for DTC. For both datasets, patients coded as receiving isotope radiation had done so in the context of treatment, rather than for diagnosis or disease monitoring.
The main outcome measures were all-cause mortality (NCDB and SEER) and thyroid cancer-specific mortality (SEER). Univariate statistics were generated for clinical, treatment, and demographic variables. Bivariate statistics were generated for RAI use and mortality, and Pearson chi-squared $P$ values determined.

Cox multivariate analyses were applied to each dataset and covariates included: receipt of RAI, T/N/M stages, age, gender, and race. Charlson Comorbidity Index (CCI) information was not available in SEER, but was added as a covariate in the NCDB analyses (reference group $\mathrm{CCI}=0$ ).

In both datasets, the same multivariate analysis was applied to 135 unique patient subgroups based on different combinations of clinical and non-clinical parameters. Covariates were identical as in the overall analyses, and collinear variables were automatically excluded from the analysis during this process. From each of the subgroup multivariate analyses, the hazard ratio (HR) for RAI use was compared to evaluate the relative impact of RAI in each subgroup.

Analyses were performed using STATA SE (Stata Corp.). All confidence intervals (CIs) are reported as 95\% CI. This study was exempt from institutional review board's review because it utilized de-identified patient information from publicly available databases.

\section{Results}

There were 199,371 patients in the NCDB and 77,187 patients in the SEER dataset that met inclusion criteria. The median ages were 44 years and 48 years, respectively. Patients were predominately female $(78.1$ and $78.5 \%$, respectively) and white (71.1 and 68.7\%, respectively). The details of demographics and other non-clinical parameters are provided in Table 1 .

\section{RAl usage}

Overall, $51.8 \%$ of NCDB patients and $46.6 \%$ of SEER patients received RAI. Use differed across various clinical and non-clinical characteristics, which is reflected in Table 1. Higher rates were seen in patients with advanced T/N/M and overall stage. Patients with M1 disease received RAI in 62.7 and $60.1 \%$ of cases (NCDB and SEER, respectively). RAI was used less often in intermediaterisk disease categories. About a quarter of patients with T1a disease received RAI (NCDB 24.5 and SEER 28.6\%). Pearson chi-squared $P$ values for RAI usage rates among subgroups ( $\mathrm{T}$ stage, $\mathrm{N}$ stage, race, etc.) were all statistically significant (Table 1). 
Table 1 Demographics, RAI usage, and mortality in patients with differentiated thyroid cancer in NCDB and SEER databases.

\begin{tabular}{|c|c|c|c|c|c|c|c|}
\hline \multirow[b]{2}{*}{ Characteristic } & \multicolumn{3}{|c|}{ NCDB } & \multicolumn{4}{|c|}{ SEER } \\
\hline & \# of patients (\%) & $\begin{array}{c}\text { Patients that } \\
\text { received } \mathrm{RAI}(\%)^{\mathrm{a}} \\
\end{array}$ & $\begin{array}{c}\text { Patients } \\
\text { that died } \\
\text { (all-cause) }(\%)^{a}\end{array}$ & $\begin{array}{c}\text { No. of } \\
\text { patients (\%) }\end{array}$ & $\begin{array}{l}\text { Patients that } \\
\text { received } \\
\text { RAI }(\%)^{\mathrm{a}}\end{array}$ & $\begin{array}{c}\text { Patients } \\
\text { that died } \\
\text { (all-cause) }(\%)^{a}\end{array}$ & $\begin{array}{c}\text { Patients that } \\
\text { died of thyroid } \\
\text { cancer }(\%)^{\text {a }} \\
\end{array}$ \\
\hline \multicolumn{8}{|l|}{ T stage } \\
\hline $1 a$ & 28,108 (15.1) & 6887 (24.5) & 347 (1.2) & 23,960 (33.9) & 6851 (28.6) & $969(4.0)$ & $43(0.2)$ \\
\hline $1 b$ & $87,570(47.2)$ & $40,413(46.2)$ & $2392(2.7)$ & $17,726(25.1)$ & 9978 (56.3) & $578(3.3)$ & $81(0.5)$ \\
\hline 2 & 32,567 (17.5) & 21,923 (67.3) & $898(2.8)$ & $14,506(20.5)$ & 8746 (60.3) & $618(4.3)$ & $192(1.3)$ \\
\hline 3 & 31,677 (17.1) & 22,977 (72.5) & $1430(4.5)$ & 9605 (13.6) & 6191 (64.5) & $571(5.9)$ & $254(2.6)$ \\
\hline 4 & $5718(3.1)$ & 4017 (70.3) & 1087 (19.0) & $4948(7.0)$ & 3323 (67.2) & $548(11.1)$ & $281(5.7)$ \\
\hline \multicolumn{8}{|l|}{ N stage } \\
\hline 0 & 141,451 (81.1) & $65,198(46.1)$ & 4101 (2.9) & 51,981 (80.9) & 23,055 (44.4) & 1860 (3.6) & $293(0.6)$ \\
\hline $1 a$ & $17,118(9.8)$ & 12,885 (75.3) & $522(3.1)$ & $4286(6.7)$ & 3124 (72.9) & $90(2.1)$ & $44(1.0)$ \\
\hline $1 b$ & $15,940(9.1)$ & $11,944(74.9)$ & 1081 (6.8) & 8029 (12.5) & 5612 (69.9) & $645(8.0)$ & $339(4.2)$ \\
\hline \multicolumn{8}{|l|}{ M stage } \\
\hline 0 & 197,232 (98.9) & 101,928 (51.7) & $6181(3.1)$ & 76,195 (98.7) & $35,372(46.4)$ & 3521 (4.6) & $724(1.0)$ \\
\hline 1 & 2139 (1.1) & 1342 (62.7) & 763 (35.7) & 992 (1.3) & $596(60.1)$ & 434 (43.6) & 299 (30.1) \\
\hline \multicolumn{8}{|l|}{ AJCC stage } \\
\hline I & $146,244(76.1)$ & $67,916(46.4)$ & 2777 (1.9) & $60,265(78.1)$ & $25,415(42.2)$ & 1993 (3.3) & $146(0.24)$ \\
\hline II & 14,706 (7.7) & $9590(65.2)$ & 663 (4.5) & 5895 (7.6) & 3426 (58.12) & $437(7.4)$ & 113 (1.9) \\
\hline III & 20,100 (10.5) & 14,385 (71.6) & 1078 (5.4) & $5379(7.0)$ & 3422 (63.6) & 373 (6.9) & $124(2.3)$ \\
\hline IV & $11,043(5.8)$ & 7886 (71.4) & 1906 (5.8) & $5648(7.3)$ & 3705 (65.6) & 1152 (20.4) & 640 (11.3) \\
\hline \multicolumn{8}{|l|}{ Sex } \\
\hline Female & $155,637(78.1)$ & $78,590(50.5)$ & $4326(2.8)$ & 60,625 (78.5) & 27,602 (45.5) & $2452(4.0)$ & $608(1.0)$ \\
\hline Male & $43,734(21.9)$ & $24,680(56.4)$ & $2618(6.0)$ & $16,562(21.5)$ & 8366 (50.5) & $1503(9.1)$ & $415(2.5)$ \\
\hline \multicolumn{8}{|l|}{ Race } \\
\hline White & $142,914(71.7)$ & 73,950 (51.7) & $5026(3.5)$ & 53,046 (68.7) & $24,190(45.6)$ & $2620(4.9)$ & $648(1.2)$ \\
\hline Black & $13,648(6.9)$ & $6181(45.3)$ & 669 (4.9) & $4478(5.8)$ & 1777 (39.7) & $363(8.1)$ & $67(1.5)$ \\
\hline Asian & 8807 (4.4) & 4724 (53.6) & $185(2.1)$ & 6056 (7.9) & 3261 (53.8) & $404(6.7)$ & $124(2.1)$ \\
\hline Hispanic & 30,711 (15.4) & $16,895(55.0)$ & $985(3.2)$ & $10,572(13.7)$ & $5397(51.1)$ & $480(4.5)$ & 159 (1.5) \\
\hline Other and unknown & 3291 (1.7) & $1520(46.2)$ & $79(2.4)$ & 3035 (3.9) & $1343(44.3)$ & $88(2.9)$ & $25(0.8)$ \\
\hline
\end{tabular}

Pearson Chi-squared test performed for each subgroup stratification (T stage, $\mathrm{N}$ stage, $\mathrm{M}$ stage, etc) in both RAl and mortality columns. $P$ values were $<0.001$ for all groups. 2010 AJCC 7 TNM criteria used for staging.

apercentages represent the \# of patients with a given characteristic (in each column) divided by the total patients with relevant data in that subgroup ('\# of patients' column).

\section{All-cause mortality (NCDB and SEER)}

All-cause mortality was $3.5 \%$ in NCDB $(n=6944)$ and $5.1 \%$ in SEER $(n=3955)$. A greater proportion of males died (NCDB: 6.0 vs $2.8 \%$ female, $P<0.001$; SEER: 9.1 vs $4.0 \%$, $P<0.001)$. Pearson chi-squared $P$ values for mortality rates among subgroups ( $\mathrm{T}$ stage, $\mathrm{N}$ stage, race, etc.) were all statistically significant (Table 1).

\section{Cancer-specific mortality (SEER)}

Thyroid cancer-specific mortality in SEER was $1.3 \%$ $(n=1023)$. Males exhibited higher mortality than females ( 2.5 vs $1.0 \%, P<0.001$ ). Asians had the highest mortality of any race, followed by blacks and Hispanics, then whites ( 2.1 vs 1.5 vs 1.5 vs $1.2 \%, P<0.001$ ). Increasing thyroid cancer-specific mortality was seen with more advanced T Stage (T1a 0.2\%, T1b 0.5\%, T2 1.3\%, T3 2.6\%, and T4 $5.7 \%, P<0.001)$. A similar trend was seen with $\mathrm{N}$ stage and
M stage (N0 0.6\%, N1a 1.0\%, N1b 4.2\%, $P<0.001$; and M0 $1.0 \%, \mathrm{M} 130.1 \%, P<0.001$ ), and overall stage (stage I $0.2 \%$, stage II $1.9 \%$, stage III $2.3 \%$, stage IV $11.3 \%, P<0.001)$.

\section{RAI and cancer-specific mortality}

Compared to those who did not receive RAI, a larger proportion of patients who received RAI ultimately died of their thyroid cancer ( 1.6 vs $1.1 \%, P<0.001)$. A detailed bivariate summary of thyroid cancer-specific mortality by RAI usage is provided in Table 2. Patients with T1a tumors who received RAI had a higher thyroid cancer-specific mortality rate than those who did not receive RAI $(0.4 \%$ (30 patients) vs $0.1 \%$ (13 patients), $P<0.001)$. This same relationship was seen for N0, M0, female, male, white, and other/unknown race subgroups (Table 2). Subgroups for which RAI use was associated with improved cancerspecific survival included T1b, T4, N1a, N1b, M1. 
Table 2 Thyroid cancer-specific mortality by RAI usage across various subgroups in SEER.

\begin{tabular}{lc}
\hline Characteristic & In patients who did not receive RAI, $\boldsymbol{n}(\%)$ \\
\cline { 1 - 2 } T stage & $13(0.1)$ \\
1a & $45(0.6)$ \\
1b & $70(1.2)$ \\
3 & $100(2.9)$ \\
4 & $119(7.3)$ \\
N stage & $127(0.4)$ \\
0 & $23(2.0)$ \\
1a & $128(5.3)$ \\
1b & \\
M stage & $300(0.7)$ \\
0 & $161(40.7)$ \\
1 & \\
AJCC stage & $80(0.2)$ \\
I & $55(2.2)$ \\
II & $53(2.7)$ \\
III & $273(14.1)$ \\
IV & \\
Sex & $291(0.9)$ \\
Female & $170(2.1)$ \\
Male & \\
Race & $275(1.0)$ \\
White & $42(1.6)$ \\
Black & $59(2.1)$ \\
Asian & $76(1.5)$ \\
Hispanic & $9(0.5)$ \\
Other and unknown &
\end{tabular}

\section{Multivariate (overall populations)}

On multivariate analysis of the overall datasets, RAI demonstrated a protective association with all-cause mortality (Supplementary Table 1, see section on supplementary data given at the end of this article, NCDB: HR 0.55 (0.52-0.58), P<0.001; SEER: HR 0.64 (0.58-0.70), $P<0.001)$. RAI had a similarly favorable association with thyroid cancer-specific mortality in the SEER dataset (Supplementary Table 1, HR 0.82 (0.69-0.98), $P=0.029)$. Multiple other factors were independently associated with decreased mortality, including female gender and lower $\mathrm{T}$ stage, with many of these findings consistent between NCDB and SEER datasets (Supplementary Table 1). The strongest negative prognosticators were advanced age (>45 years), M1 disease, and presence of nodal metastases.

\section{Multivariate (subgroups)}

Applying the same multivariate analysis to 135 unique patient subgroups, the use of RAI was significantly associated with improved all-cause mortality outcomes in 132 of the NCDB subgroups and 109 subgroups in SEER. There were statistically significant results for 37 subgroups of the SEER thyroid cancer-specific analyses. An overview

\begin{tabular}{|c|c|}
\hline In patients who received $\mathbf{R A I}, \boldsymbol{n}(\%)$ & Pearson chi-squared $\boldsymbol{P}$ value \\
\hline $30(0.4)$ & $<0.001$ \\
\hline $36(0.4)$ & 0.03 \\
\hline $122(1.4)$ & 0.35 \\
\hline $154(2.5)$ & 0.20 \\
\hline $162(4.9)$ & $<0.001$ \\
\hline $166(0.7)$ & $<0.001$ \\
\hline $21(0.7)$ & $<0.001$ \\
\hline $211(3.8)$ & 0.002 \\
\hline $424(1.2)$ & $<0.001$ \\
\hline $138(23.2)$ & $<0.001$ \\
\hline $66(0.3)$ & 0.46 \\
\hline $58(1.7)$ & 0.14 \\
\hline $71(2.1)$ & 0.14 \\
\hline 367 (9.9) & $<0.001$ \\
\hline $317(1.2)$ & 0.001 \\
\hline 245 (2.9) & $<0.001$ \\
\hline $373(1.5)$ & $<0.001$ \\
\hline $25(1.4)$ & 0.69 \\
\hline $65(2.0)$ & 0.75 \\
\hline $83(1.5)$ & 0.77 \\
\hline $16(1.2)$ & 0.046 \\
\hline
\end{tabular}

of the RAI HRs from the broad subgroup categories is provided in Table 3, and a full report of all 135 subgroups is provided in Supplementary Table 2. In these tables, each row reports the HR for receiving RAI.

Patients with high-risk DTC (M1, stage 4, T4, N+) showed improved survival related to RAI. For example, patients in the NCDB with M1 disease who received RAI were $49 \%$ less likely to die than their M1 counterparts who did not receive RAI (Table 3). Survival was improved by $61 \%$ in this same subgroup in SEER. Regarding cancer-specific mortality, M1 patients in SEER were 63\% less likely to die of their thyroid cancer after receiving RAI treatment.

Improvements in all-cause mortality with RAI usage ranged from HR 0.61 to 0.42 in NCDB and 0.79 to 0.39 in SEER, spanning from high-risk down to intermediate-risk and low-risk groups. The only group that showed increased disease-specific mortality with RAI use were T1a patients (HR 7.89 (2.54-24.55), $P<0.001$ ).

\section{Discussion}

It is well established that RAI is a key component in the management of DTC. The effect of this treatment has not c) 2019 Society for Endocrinology Published by Bioscientifica Ltd. Printed in Great Britain 


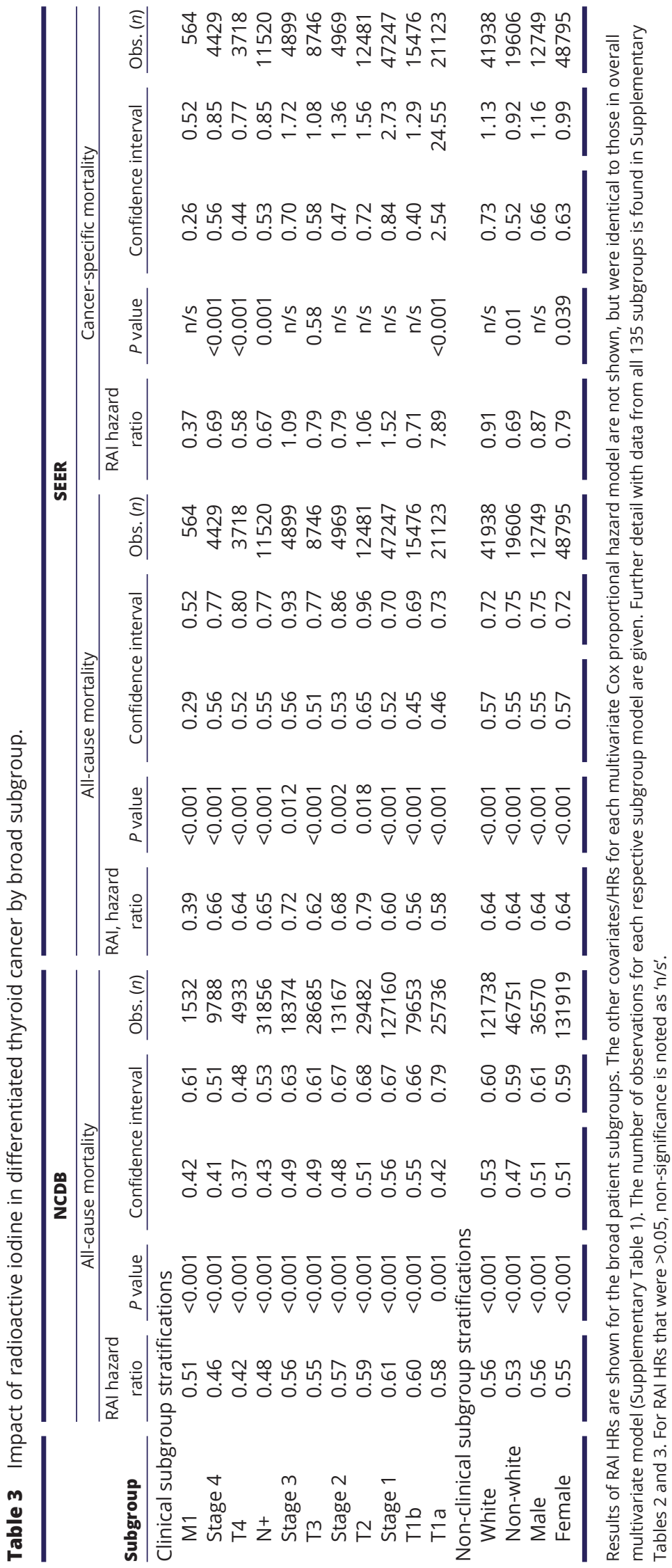


been compared across risk groups in a single study. Using the two largest cancer databases in the United States, we found RAI to be broadly efficacious. Using all-cause and cancer-specific mortality outcomes, we characterized the impact that RAI had on specific patient groups.

It is important to note that, due to the retrospective nature of our study, the findings should be considered as preliminary evidence and not an establishment of quantification of treatment effect. National observational databases like SEER and NCDB are useful in evaluating low-probability events (like mortality in thyroid cancer) because they provide large sample sizes. Unfortunately, they lack key information regarding the extent of surgery, RAI dose, histologic subtype, genetic mutations, and other tumor or nodal factors that may have influenced the decision to give RAI. Furthermore, controlling for multiple covariates does not guarantee an absence of confounders and collinear variables with complex interactions. Disease recurrence is another outcome not attainable from these large datasets that is particularly relevant in thyroid cancer. At present, there are at least two trials enrolling patients to evaluate the impact of RAI in low-risk thyroid cancer patients (ClinicalTrials.gov: NCT01837745, NCT01398085), and we will await those results for more robust evidence.

Despite the shortcomings of this study design, valuable insights can be gained from evaluating the two largest national cancer databases in the United States. The current state of our understanding of RAI efficacy is primarily based on institutional experience and smaller database studies. In a prior SEER analysis of 14,545 patients, survival was not affected by RAI (Podnos et al. 2007); the current study of more than five times the number of patients showed a clear benefit for the overall population of DTC patients. This broad multivariate approach (Supplementary Table 1) is useful in that it describes the risk factors for mortality. Our subgroup analyses delve into the impact of RAI in specific patient populations (Table 3). In these subgroups, our findings largely align with current consensus recommendations and with multiple smaller studies that sought to define the impact of RAI in specific DTC populations.

When reviewing the subgroup analyses, it should be emphasized that the HRs cannot be directly compared between rows (subgroups), nor across mortality outcomes (Table 3 and Supplementary Table 2). Each HR denotes the impact of RAI on mortality risk within that specific subgroup (row). For example, NCDB patients demonstrated a decreased risk of mortality in both $\mathrm{M} 1$ and $\mathrm{T} 2$ subgroups
(HR 0.51 and 0.59, respectively), but each of these subgroups carried a different baseline hazard mortality rate (35.7 vs $2.8 \%$, Table 1 ). A direct HR comparison would incorrectly conclude that RAI is $8 \%$ more beneficial in patients with $\mathrm{M} 1$ disease than in those with $\mathrm{T} 2$ disease. Similarly, because the $95 \%$ CI in each of these subgroups overlap, one should avoid concluding that the impact of RAI does not differ between the subgroups. Properly framed, RAI use in M1 patients was associated with a $49 \%$ improvement in survival relative to the $35.7 \%$ mortality rate in this subgroup. Similarly, RAI use in T2 patients was associated with a $41 \%$ decrease in mortality risk, with a baseline mortality of only $2.8 \%$ in that subgroup.

According to ATA risk stratification, high-risk DTC includes cases of macroscopic extension of tumor into adjacent tissues, incomplete tumor resection, distant metastases, bulky regional nodal metastases, and aggressive histology (Haugen et al. 2016). In agreement with current guidelines (Haugen et al. 2016), our study shows a survival advantage in patients with advanced disease that are treated with RAI. The benefit of adjuvant RAI for high-risk DTC is undisputed, and therapy is routinely recommended in this population. Notably, both the NCDB and the SEER datasets indicate a likely underutilization of RAI in high-risk patients, especially those with M1 disease. Based on the available information it is not possible to determine why a patient in this subset may not have received RAI. However, this analysis highlights potential care discrepancies and deviation from guidelines.

Evidence surrounding the use of RAI in patients with intermediate-risk DTC is less robust, and there is no consensus as to the risk/benefit ratio. Characteristics that make a patient intermediate-risk include microscopic tumor invasion into adjacent tissues, regional disease on RAI uptake scan following treatment, aggressive histology, vascular invasion, $>5$ positive nodes, and multifocal carcinoma with extra-thyroidal spread and BRAF mutation (Haugen et al. 2016). In our study, intermediate-risk subgroups with positive nodes and $\mathrm{T} \geq 3$ disease experienced a mortality benefit with RAI use. A prior NCDB study showed a favorable association between post-operative RAI and survival in intermediaterisk DTC patients, (Ruel et al. 2015) and several other studies have also demonstrated improved outcomes in this risk group.(Creach et al. 2012, Kazaure et al. 2012) However, significant controversy exists (Hay et al. 2002, Kim et al. 2013) and a recent systematic review highlights the conflicting data regarding the utility of RAI for intermediate-risk patients - 11 studies observed some 
benefit from RAI in reducing recurrence, while 13 showed no benefit. Survival outcomes were not addressed in this review (Lamartina et al. 2015). Overall, RAI should be considered for intermediate-risk patients (Haugen et al. 2016), but the specific patient and tumor characteristics must be factored into the decision-making process.

Low-risk DTC patients are those whose disease is small, confined to the thyroid, without aggressive histology, with complete resection, and without regional spread (Haugen et al. 2016). There is a mounting evidence suggesting that the use of RAI may constitute unnecessary treatment in low-risk patients (Schvartz et al. 2012, Kim et al. 2013, Carhill et al. 2015, Lamartina et al. 2015, Lamartina \& Cooper 2015), and it is not currently recommended in this group (Haugen et al. 2016). Importantly, the use of evidence-based guidelines has been shown to reduce RAI treatment in patients with low-risk DTC (Sacks et al. 2015). It should also be noted that current ATA guidelines discuss active surveillance as an alternative to immediate surgery for select patients with low-risk disease (Haugen et al. 2016). Our study showed an overall survival benefit for RAI use in low-risk patients. This should be interpreted in the context of exceptionally low baseline mortality rates, and with the realization that recurrence is the more salient outcome in this population. Our finding of increased risk for cancerspecific mortality in T1a subgroups was unexpected, though other studies of early-stage patients have reported similar negative associations between RAI and overall survival (Jonklaas et al. 2006) and recurrent disease (Sacks et al. 2015). The tumor or patient characteristics that may have led to the decision to treat with RAI are not discernible and could reveal more aggressive or concerning features among this group.

Based on NCDB data, RAI use in the United States rose between 1990 and 2008, and nearly a third of patients with T1a disease received treatment (Haymart et al. 2011). A prior SEER study from 1983 to 2009 showed that the proportion of low-risk DTC patients receiving RAI increased from 8 to 31\% (Marti et al. 2015a). A variety of patient, provider, hospital, regional, and system factors have been shown to affect RAI usage (Haymart et al. 2011, 2013a,b, Marti et al. 2015a), and there is evidence of racial and socioeconomic inconsistencies (Goffredo et al. 2015). With the incidence of thyroid cancer on the rise, and more than $85 \%$ of newly detected DTCs being smaller than $2 \mathrm{~cm}$ (Davies \& Welch 2006), a significant proportion of patients and clinicians are left with uncertainty when considering post-thyroidectomy therapy. The administration of RAI comes with financial cost (Pace-Asciak et al. 2007, Goffredo et al. 2015) and risks that range from salivary gland dysfunction (Van Nostrand 2009 ) to second primary malignancies (Sawka et al. 2009, Iyer et al. 2011, Marti et al. 2015b, Correa et al. 2016, Teng et al. 2016). The role of RAI in DTC is evolving, and, in an era of increasingly personalized medicine, it is critical to consider the individual risk factors of each patient. Both individual patients and the healthcare system stand to gain from evidence-based usage of RAI.

This is the largest study to date analyzing the effect of RAI on all-cause and cancer-specific mortality in patients with DTC. The sample size and multivariate analyses of our study overcome many of the weaknesses of previous work that reported conflicting associations with RAI usage. Broadly, RAI appears to be efficacious, with a graded effect in various subgroups. Our results affirm the current ATA recommendation for RAI in high-risk DTC, and for caseby-case consideration in intermediate-risk populations. It also highlights potential underutilization and lack of adherence to guidelines in patients with distant metastatic disease. In patients with T1a tumors, we demonstrated an unanticipated negative association with cancer-specific mortality. This may be related to factors beyond the ability of a database study to ascertain and should not be considered a causative relationship. However, it does emphasize that the decision to treat with RAI should be made in an informed manner and that future prospective study must take into account dynamic risk assessment in addition to initial stratification.

\section{Supplementary data}

This is linked to the online version of the paper at https://doi.org/10.1530/ ERC-19-0292.

\section{Declaration of interest}

The authors declare that there is no conflict of interest that could be perceived as prejudicing the impartiality of the research reported.

\section{Funding}

This research was supported by a National Institutes of Health Ruth Kirschstein National Research Service Award (NRSA) Institutional Research Training Grant (T32 DC000128) to R K O.

\section{References}

Carhill AA, Litofsky DR, Ross DS, Jonklaas J, Cooper DS, Brierley JD, Ladenson PW, Ain KB, Fein HG, Haugen BR, et al. 2015 Long-term outcomes following therapy in differentiated thyroid carcinoma: NTCTCS Registry Analysis 1987-2012. Journal of Clinical Endocrinology and Metabolism 100 3270-3279. (https://doi. org/10.1210/JC.2015-1346)
(C) 2019 Society for Endocrinology Published by Bioscientifica Ltd. Printed in Great Britain 
Correa NL, de Sa LV \& Corbo R 2016 Estimation of second primary cancer risk after treatment with radioactive iodine for differentiated thyroid carcinoma. Thyroid 27 261-270. (https://doi.org/10.1089/ thy.2016.0266)

Creach KM, Siegel BA, Nussenbaum B \& Grigsby PW 2012 Radioactive iodine therapy decreases recurrence in thyroid papillary microcarcinoma. ISRN Endocrinology 2012 816386. (https://doi. org/10.5402/2012/816386)

Davies L \& Welch HG 2006 Increasing incidence of thyroid cancer in the United States, 1973-2002. JAMA 295 2164-2167. (https://doi. org/10.1001/jama.295.18.2164)

Goffredo P, Thomas SM, Dinan MA, Perkins JM, Roman SA \& Sosa JA 2015 Patterns of use and cost for inappropriate radioactive iodine treatment for thyroid cancer in the United States: use and misuse. JAMA Internal Medicine 175 638-640. (https://doi.org/10.1001/ jamainternmed.2014.8020)

Haugen BR, Alexander EK, Bible KC, Doherty GM, Mandel SJ, Nikiforov YE, Pacini F, Randolph GW, Sawka AM, Schlumberger M, et al. 20162015 American Thyroid Association management guidelines for adult patients with thyroid nodules and differentiated thyroid cancer: the American Thyroid Association Guidelines Task Force on thyroid nodules and differentiated thyroid cancer. Thyroid 26 1-133. (https://doi.org/10.1089/thy.2015.0020)

Hay ID, Thompson GB, Grant CS, Bergstralh EJ, Dvorak CE, Gorman CA, Maurer MS, McIver B, Mullan BP, Oberg AL, et al. 2002 Papillary thyroid carcinoma managed at the Mayo Clinic during six decades (1940-1999): temporal trends in initial therapy and long-term outcome in 2444 consecutively treated patients. World Journal of Surgery 26 879-885. (https://doi.org/10.1007/ s00268-002-6612-1)

Haymart MR, Banerjee M, Stewart AK, Koenig RJ, Birkmeyer JD \& Griggs JJ 2011 Use of radioactive iodine for thyroid cancer. JAMA 306 721-728. (https://doi.org/10.1001/jama.2011.1139)

Haymart MR, Banerjee M, Yang D, Stewart AK, Koenig RJ \& Griggs JJ $2013 a$ The role of clinicians in determining radioactive iodine use for low-risk thyroid cancer. Cancer 119 259-265. (https://doi. org/10.1002/cncr.27721)

Haymart MR, Banerjee M, Yang D, Stewart AK, Sisson JC, Koenig RJ, Doherty GM \& Griggs JJ 2013 $b$ Variation in the management of thyroid cancer. Journal of Clinical Endocrinology and Metabolism 98 2001-2008. (https://doi.org/10.1210/jc.2012-3355)

Iyer NG, Morris LG, Tuttle RM, Shaha AR \& Ganly I 2011 Rising incidence of second cancers in patients with low-risk (T1N0) thyroid cancer who receive radioactive iodine therapy. Cancer 1174439 4446. (https://doi.org/10.1002/cncr.26070)

Jonklaas J, Sarlis NJ, Litofsky D, Ain KB, Bigos ST, Brierley JD, Cooper DS, Haugen BR, Ladenson PW, Magner J, et al. 2006 Outcomes of patients with differentiated thyroid carcinoma following initial therapy. Thyroid 16 1229-1242. (https://doi org/10.1089/thy.2006.16.1229)

Kazaure HS, Roman SA \& Sosa JA 2012 Aggressive variants of papillary thyroid cancer: incidence, characteristics and predictors of survival among 43,738 patients. Annals of Surgical Oncology 19 1874-1880. (https://doi.org/10.1245/s10434-011-2129-x)

Kim HJ, Kim NK, Choi JH, Kim SW, Jin SM, Suh S, Bae JC, Min YK, Chung JH \& Kim SW 2013 Radioactive iodine ablation does not prevent recurrences in patients with papillary thyroid microcarcinoma. Clinical Endocrinology 78 614-620. (https://doi. org/10.1111/cen.12034)
Lamartina L \& Cooper DS 2015 Radioiodine remnant ablation in lowrisk differentiated thyroid cancer: the 'con' point of view. Endocrine 50 67-71. (https://doi.org/10.1007/s12020-014-0523-4)

Lamartina L, Durante C, Filetti S \& Cooper DS 2015 Low-risk differentiated thyroid cancer and radioiodine remnant ablation: a systematic review of the literature. Journal of Clinical Endocrinology and Metabolism 100 1748-1761. (https://doi.org/10.1210/jc.2014-3882)

Marti JL, Davies L, Haymart MR, Roman BR, Tuttle RM \& Morris LG $2015 a$ Inappropriate use of radioactive iodine for low-risk papillary thyroid cancer is most common in regions with poor access to healthcare. Thyroid 25 865-866. (https://doi.org/10.1089/ thy.2015.0112)

Marti JL, Jain KS \& Morris LGT $2015 b$ Increased risk of second primary malignancy in pediatric and young adult patients treated with radioactive iodine for differentiated thyroid cancer. Thyroid $\mathbf{2 5} 681$ 687. (https://doi.org/10.1089/thy.2015.0067)

Pace-Asciak PZ, Payne RJ, Eski SJ, Walfish P, Damani M \& Freeman JL 2007 Cost savings of patients with a MACIS score lower than 6 when radioactive iodine is not given. Archives of Otolaryngology: Head and Neck Surgery 133 870-873. (https://doi.org/10.1001/archotol.133.9.870)

Podnos YD, Smith DD, Wagman LD \& Ellenhorn JD 2007 Survival in patients with papillary thyroid cancer is not affected by the use of radioactive isotope. Journal of Surgical Oncology 96 3-7. (https://doi. org/10.1002/jso.20656)

Ruel E, Thomas S, Dinan M, Perkins JM, Roman SA \& Sosa JA 2015 Adjuvant radioactive iodine therapy is associated with improved survival for patients with intermediate-risk papillary thyroid cancer. Journal of Clinical Endocrinology and Metabolism 100 1529-1536. (https://doi.org/10.1210/jc.2014-4332)

Sacks W, Wong RM, Bresee C \& Braunstein GD 2015 Use of evidencebased guidelines reduces radioactive iodine treatment in patients with low-risk differentiated thyroid cancer. Thyroid 25 377-385. (https://doi.org/10.1089/thy.2014.0298)

Sawka AM, Thabane L, Parlea L, Ibrahim-Zada I, Tsang RW, Brierley JD, Straus S, Ezzat S \& Goldstein DP 2009 Second primary malignancy risk after radioactive iodine treatment for thyroid cancer: a systematic review and meta-analysis. Thyroid 19 451-457. (https:// doi.org/10.1089/thy.2008.0392)

Schvartz C, Bonnetain F, Dabakuyo S, Gauthier M, Cueff A, Fieffe S, Pochart JM, Cochet I, Crevisy E, Dalac A, et al. 2012 Impact on overall survival of radioactive iodine in low-risk differentiated thyroid cancer patients. Journal of Clinical Endocrinology and Metabolism 97 1526-1535. (https://doi.org/10.1210/jc.2011-2512)

Sherman SI 2003 Thyroid carcinoma. Lancet 361 501-511. (https://doi. org/10.1016/s0140-6736(03)12488-9)

Siegel RL, Miller KD \& Jemal A 2017 Cancer statistics, 2017. CA: A Cancer Journal for Clinicians 67 7-30. (https://doi.org/10.3322/caac.21387)

Teng CJ, Hu YW, Chen SC, Yeh CM, Chiang HL, Chen TJ \& Liu CJ 2016 Use of radioactive iodine for thyroid cancer and risk of second primary malignancy: a nationwide population-based study. Journal of the National Cancer Institute 108 djv314. (https://doi.org/10.1093/ jnci/djv314)

Vaccarella S, Franceschi S, Bray F, Wild CP, Plummer M \& Dal Maso L 2016 Worldwide thyroid-cancer epidemic? The increasing impact of overdiagnosis. New England Journal of Medicine 375 614-617. (https:// doi.org/10.1056/NEJMp1604412)

Van Nostrand D 2009 The benefits and risks of I-131 therapy in patients with well-differentiated thyroid cancer. Thyroid 19 1381-1391. (https://doi.org/10.1089/thy.2009.1611)

Received in final form 13 August 2019

Accepted 23 August 2019

Accepted Preprint published online 23 August 2019 https://erc.bioscientifica.com https://doi.org/10.1530/ERC-19-0292 (c) 2019 Society for Endocrinology Published by Bioscientifica Ltd. Printed in Great Britain 